\title{
Halving lines and perfect cross-matchings
}

\author{
János Pach ${ }^{1}$ and József Solymosi ${ }^{2}$ \\ Mathematical Institute of the \\ Hungarian Academy of Sciences \\ H-1364 Budapest, P.O.B. 127
}

\begin{abstract}
It is shown that a set $P$ of $2 n$ points in general position in the plane admits a perfect matching with pairwise crossing segments if and only if it has precisely $n$ halving lines. As a consequence, one can give a $O(n \log n)$-time algorithm which decides whether there exists such a matching in $P$ and, if so, finds it.
\end{abstract}

\section{Preliminaries}

Let $P=\left\{p_{1}, p_{2}, \ldots, p_{2 n}\right\}$ be a set of $2 n$ points in the plane in general position, i.e., no three points are collinear. A line $p_{i} p_{j}$ is said to be a halving line of $P$ if both open half-planes bounded by $p_{i} p_{j}$ contain precisely $n-1$ points. The number of halving lines of $P$ is denoted by $h(P)$.

Taking an arbitrary line through any point of $P$ and turning it around by at most 180 degrees, it always arrives at a position where it becomes a halving line. Thus, we have $h(P) \geq n$, and equality holds, e.g., when $P$ is the vertex set of a convex $2 n$-gon.

It is an intriguing open problem to determine the asymptotic behavior of $h(n)=\max _{P} h(P)$, where the maximum is taken over all $2 n$-element sets in general position in the plane. It is known that

$$
c_{1} n \log n \leq h(n) \leq c_{2} n^{4 / 3}
$$

for suitable constants $c_{1}, c_{2}>0$ (see [L], [EL], [D]). This function plays an important role in the analysis of many algorithms in computational geometry (cf. $[\mathrm{E}]$ ).

\footnotetext{
${ }^{1}$ Supported by NSF grant CR-94-24398, PSC-CUNY Research Award 663472, and OTKA-T-020914.

${ }^{2}$ Supported by the DIMATIA Center, Prague.
} 
We say that two segments cross if they have an interior point in common. Let $c(P)$ denote the maximum number of pairwise crossing segments $\overline{p_{i} p_{j}}$ whose endpoints belong to $P$. Obviously, $c(P) \leq n$ holds for every $2 n$ element set $P$. If $c(P)=n$, we say that $P$ has a perfect cross-matching. This is the case, for example, when $P$ is the vertex set of a convex $2 n$-gon.

Let $c(n)=\min _{P} c(P)$, where the minimum is taken over all $2 n$-element sets in general position in the plane. We have

$$
c(n) \geq c_{3} \sqrt{n},
$$

for some positive constant $c_{3}$, but there is no sublinear upper bound known for $c(n)$ (see $[\mathrm{A}],[\mathrm{P}])$. In fact, in [A] it was shown that every $2 n$-element set in general position has an at least $c_{3} \sqrt{n}$-element subset which not only admits a perfect cross-matching, but also satisfies a much stronger condition. In this strong sense the result is best possible [V]. It looked difficult to improve the lower bound on $c(n)$, because we had no good characterization of perfectly cross-matchable sets.

The aim of this note is to give such a good characterization and to design an efficient algorithm which decides whether a set admits a perfect crossmatching.

\section{Characterization of perfectly cross-matchable sets}

In this section, we would like to point out a simple relation between $c(P)$ and $h(P)$ : the first quantity attains its maximum if and only if the second attains its minimum. More precisely, we have the following.

Theorem 1. A set of $2 n$ points in general position in the plane admits a perfect cross-matching if and only if it has precisely $n$ halving lines.

Proof: Suppose first that $P=\left\{p_{1}, p_{2}, \ldots, p_{2 n}\right\}$ has a perfect cross-matching (i.e., $n$ pairwise crossing segments) $\overline{p_{2 i-1} p_{2 i}}, 1 \leq i \leq n$. The extension of each of these segments is a halving line, because each of them separates the two endpoints of all other segments $\overline{p_{2 i-1} p_{2 i}}$. We will show that $P$ has no other halving lines.

Assume, in order to obtain a contradiction, that (say) $p_{1} p_{3}$ is also a halving line. We may suppose without loss of generality that $p_{1} p_{2}$ is horizontal, $p_{2}$ is to the right of $p_{1}$, and that $p_{2 i}$ is below and $p_{2 i-1}$ is above $p_{1} p_{2}$, for every $2 \leq i \leq n$. Since each segment $\overline{p_{2 i-1} p_{2 i}} \quad(3 \leq i \leq n)$ has to cross $\overline{p_{1} p_{2}}$, if $\overline{p_{2 i-1} p_{2 i}}$ has an endpoint to the left of $p_{1} p_{3}$, then its other endpoint must lie to the right of $p_{1} p_{3}$. However, both $p_{2}$ and $p_{4}$ are on the right-hand 
side of $p_{1} p_{3}$. This implies that the number of points to the right of $p_{1} p_{3}$ exceeds by at least 2 the number of points to the left of it, contradicting our assumption that $p_{1} p_{3}$ is a halving line.

Suppose next that $P$ has precisely $n$ halving lines. Since there is at least one halving line through every point $p_{k}$, we obtain that there must be exactly one. Thus, we can assume without loss of generality that the complete list of halving lines is $p_{2 i-1} p_{2 i} \quad(1 \leq i \leq n)$. We will show that the segments $\overline{p_{2 i-1} p_{2 i}}(1 \leq i \leq n)$ are pairwise crossing.

Assume, for contradiction, that $\overline{p_{1} p_{2}}$ and $\overline{p_{3} p_{4}}$ have no interior point in common. By renumbering the points if necessary, we may also suppose that $p_{1} p_{2}$ is horizontal, $p_{2}$ is to the right of $p_{1}, \overline{p_{3} p_{4}}$ is entirely above the line $p_{1} p_{2}$, and that $p_{3}$ is closer to $p_{1} p_{2}$ than $p_{4}$ is. Notice that a minor counterclockwise turn around $p_{3}$ will bring the line $\ell=p_{3} p_{4}$ into a position, where there are precisely $n$ points on its right-hand side. (Indeed, $p_{4}$ will be added to the set of points to the right of $p_{3} p_{4}$.) If we continue to turn $\ell$ around $p_{3}$ in the counter-clockwise direction, we arrive at a position where $\ell$ becomes parallel to $p_{1} p_{2}$, i.e., it becomes horizontal. At that moment, there are at most $n-2$ points above $\ell$ (these points form a subset of the set of all points different from $p_{3}$ which lie above the halving line $p_{1} p_{2}$ ). Hence, there is an intermediate position $\ell=p_{3} p_{k}$ for some $k \neq 4$, in which the number of points on the right-hand side of $\ell$ is precisely $n-1$. This means that $p_{3} p_{k}$ is a halving line which does not appear in the complete list of halving lines, $p_{2 i-1} p_{2 i}(1 \leq i \leq n)$. Contradiction.

Actually, this argument also yields the uniqueness of the perfect crossmatching.

Theorem 2. Any set of points in general position in the plane admits at most one perfect cross-matching.

Proof: As we have shown, every perfect cross-matching of $P$ consists of exactly those segments between two points of $P$, whose extensions are halving lines of $P$.

\section{Algorithm}

The above characterization of perfectly cross-matchable sets allows us to design an $O(n \log n)$-time algoritm which decides whether a set of $2 n$ points satisfies this property and, if so, finds a perfect cross-matching for it.

Let $P$ be a $2 n$-element point set in general position in the plane, which is the union of two $n$-element sets, $P_{1}$ and $P_{2}$, separated by a straight line (say, by the $y$-axis). For any non-vertical line $\ell$, let $P_{i}\left(\ell^{+}\right)$(resp. $P_{i}\left(\ell^{-}\right)$) 
denote the set of points in $P_{i}$ lying above (resp. below) $\ell$. A line $\ell$ not passing through any point of $P$ is called a ham-sandwich cut for $P$, if

$$
\left|P_{1}\left(\ell^{+}\right)\right|=\left|P_{2}\left(\ell^{-}\right)\right|=\lfloor n / 2\rfloor .
$$

It was shown by Megiddo [M] that one can always find such a line $\ell$ in $O(n)$ steps (see also $[\mathrm{LM}]$ ).

Any matching of $P$ that has a segment to the left of the $y$-axis, has another one to the right of it, and these two segments cannot cross. Thus, if there exists a perfect cross-matching for $P$, then all of its segments must cross the $y$-axis and, similarly, they must also cross the ham-sandwich cut $\ell$. Consequently, a perfect cross-matching $M$ for $P$ is the union of a perfect cross-matching $M_{1}$ for $P_{1}\left(\ell^{+}\right) \cup P_{2}\left(\ell^{-}\right)$and a perfect cross-matching $M_{2}$ for $P_{1}\left(\ell^{-}\right) \cup P_{2}\left(\ell^{+}\right)$. Let $M_{i}^{+}$and $M_{i}^{-}$denote the upper envelope and the lower envelope (i.e., the pointwise maximum and pointwise minimum) of the lines supporting the segments of $M_{i}$, respectively $(i=1,2)$. Clearly, $M_{i}^{+}$and $M_{i}^{-}$are unbounded convex polygonal paths, with at most $\lfloor n / 2\rfloor$ vertices each. (See Figure 1.)

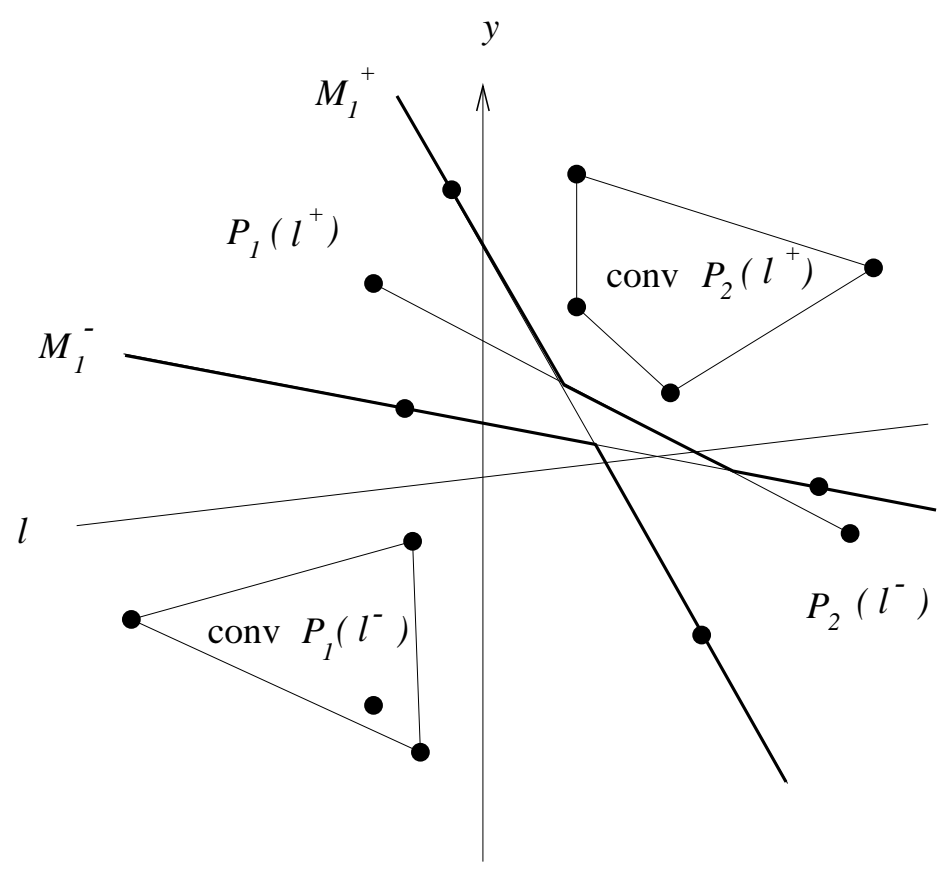

Figure 1.

We need the following corollary of Theorem 2 . 
Claim. The set $P$ admits a perfect cross-matching $M$ if and only if the following conditions are satisfied.

(1) $P_{1}\left(\ell^{+}\right) \cup P_{2}\left(\ell^{-}\right)$admits a perfect cross-matching $M_{1}$ and $P_{1}\left(\ell^{-}\right) \cup$ $P_{2}\left(\ell^{+}\right)$admits a perfect cross-matching $M_{2}$.

(2) The convex hull conv $P_{2}\left(\ell^{+}\right)$is above the polygonal path $M_{1}^{+}$, and conv $P_{1}\left(\ell^{-}\right)$is below $M_{1}^{-}$. Similarly, conv $P_{1}\left(\ell^{+}\right)$is above $M_{2}^{+}$, and conv $P_{2}\left(\ell^{-}\right)$ is below $M_{2}^{-}$.

Then, we have $M=M_{1} \cup M_{2}$.

Proof. We have seen before that if $P$ admits a perfect cross-matching $M$, then it satisfies condition (1) and $M=M_{1} \cup M_{2}$ holds. By Theorem 2, $M_{1}$ and $M_{2}$ are uniquely determined. To see that (2) is necessary, too, assume that (say) $P_{2}\left(\ell^{+}\right)$has a point $p$ below $M_{1}^{+}$. Then $p$ lies below the supporting line of at least one segment $\overline{q q^{\prime}} \in M_{1}$. Let $p^{\prime}$ denote the element of $P_{1}\left(\ell^{-}\right)$ connected to $p$ in $M_{2}$. Then $\overline{p p^{\prime}} \cap \overline{q q^{\prime}}=\emptyset$, contradicting our assumption that any two segments of $M$ cross.

Suppose next that conditions (1) and (2) are satisfied. Then $M=M_{1} \cup$ $M_{2}$ is a perfect cross-matching for $P$. Indeed, if there were two disjoint segments, $\overline{p p^{\prime}} \in M_{1}$ and $\overline{q q^{\prime}} \in M_{2}$, such that (say) $\overline{q q^{\prime}}$ is below (resp. above) the line $p p^{\prime}$, then $\operatorname{conv} P_{2}\left(\ell^{+}\right)$would not lie above the polygonal path $M_{1}^{+}$(resp. conv $P_{1}\left(\ell^{-}\right)$would not lie below $M_{1}^{-}$), contradicting condition (2).

Let $M^{+}$and $M^{-}$denote the upper and the lower envelope of all lines supporting the segments of $M=M_{1} \cup M_{2}$, respectively. Clearly, $M^{+}$can be obtained as the upper envelope of $M_{1}^{+}$and $M_{2}^{+}$, and $M^{-}$can be obtained as the lower envelope of $M_{1}^{-}$and $M_{2}^{-}$.

It is well known that one can compute the union and the intersection of two convex polygons of at most $n$ sides in time $O(n)([\mathrm{PH}],[\mathrm{S}])$. Thus, if we know $\operatorname{conv} P_{i}\left(\ell^{+}\right), \operatorname{conv} P_{i}\left(\ell^{-}\right), M_{i}^{+}$, and $M_{i}^{-}$for $i=1,2$, then in linear time we can determine conv $P_{i}(i=1,2), M^{+}$and $M^{-}$. If any of the conditions of the Claim is not satisfied, we conclude that $P$ does not admit a perfect cross-matching.

So one can use a divide-and-conquer algorithm to decide whether $P=$ $P_{1} \cup P_{2}$ admits a perfect cross-matching and, if yes, to compute it simultaneously with $\operatorname{conv} P_{i} \quad(i=1,2), M^{+}$and $M^{-}$. At each stage it takes linear time to find a ham-sandwich cut $\ell$ and to do the merge step.

We obtained the following.

Theorem 3. There is an $O(n \log n)$ time, $O(n)$ space algorithm which decides whether a set of $2 n$ points in general position in the plane admits a perfect cross-matching and, if so, computes it. 
Clearly, any decision tree that determines the perfect cross-matching of a planar point set of $2 n$ points (if it exists) has height $\Omega(n \log n)$. In this sense Theorem 3 is asymptotically tight.

\section{References}

[A] B. Aronov, P. Erdős, W. Goddard, D.J. Kleitman, M. Klugerman, J. Pach, and L.J. Schulman, Crossing families, Combinatorica 14 (1994), 127-134.

[D] T. Dey, Improved bounds for $k$-sets, Discrete and Computational Geometry, to appear.

[E] H. Edelsbrunner, Algorithms in Combinatorial Geometry, SpringerVerlag, New York, 1987.

[EL] P. Erdős, L. Lovász, A. Simmons, and E. Straus, Dissection graphs of planar point sets, in: A Survey of Combinatorial Theory (G. Srivastava, ed.), North-Holland, Amsterdam, 1973, 139-149.

[LM] Chi-Yuan Lo, J. Matoušek, and W. Steiger, Algorithms for hamsandwich cuts, Discrete and Computational Geometry 11 (1994), $433-452$.

[L] L. Lovász, On the number of halving lines, Annales Universitatis Scientarium Budapest, Eötvös, Sectio Mathematica 14 (1971), 107108.

[M] N. Megiddo, Partitioning with two lines in the plane, J. Algorithms 6 (1985), 430-433.

[P] J. Pach and P.K. Agarwal, Combinatorial Geometry, J. Wiley \& Sons, New York, 1995.

[PH] F.P. Preparata and S.J. Hong, Convex hulls of finite sets of points in two and three dimensions, Communications ACM 20 (1977), 87-93.

[S] M.I. Shamos, Computational Geometry, Ph.D. Thesis, UMI No. 7819047, Yale University, New Haven, CT, 1978.

[V] P. Valtr, On mutually avoiding sets, in: The Mathematics of Paul Erdős, II (R. L. Graham and J. Nešetřil, eds.), Algorithms and Combin. 14, Springer, Berlin, 1997, 324-332. 